\title{
Digital photography in orthopaedics: Ethical considerations
}

\author{
P O'Farrell BVSc, MBBCh \\ N Ferreira BSc, MBChB, FC Orth(SA), MMed Orth, PhD \\ University of KwaZulu-Natal \\ Corresponding author: \\ Dr Peter O'Farrell \\ Department of Orthopaedic Surgery \\ Grey's Hospital \\ Nelson R. Mandela School of Medicine \\ University of KwaZulu-Natal \\ 3201 Pietermaritzburg \\ South Africa \\ Tel: +2733 8973000 \\ Email: peterthevet@gmail.com
}

Department of Orthopaedic Surgery, Grey's Hospital, Nelson R. Mandela School of Medicine,

\begin{abstract}
Orthopaedic trauma surgery manages soft tissue damage with underlying bony injuries. As a part of the management, many surgeons use photographs to digitally capture the state of the soft tissues in order to plan further surgery. This area relies solely on individuals to regulate as to what is photographed and what happens to these images once they have served their purpose. This article reports a survey of orthopaedic surgeons in South Africa, both in state and private practice, regarding their current views and habits when obtaining digital images of clinical cases. The results demonstrate that digital images form an integral part of orthopaedic practice and are used to aid handovers, referrals and communication with patients and colleagues. The problematic areas identified were those relating to the obtaining and documenting of consent and the secure storage of images.
\end{abstract}

Key words: photography, cellphone, multimedia, orthopaedic surgery, patient privacy

http:/ / dx.doi.org/10.17159/2309-8309/2016/v15n1a12

\section{Introduction}

Advances in technology over the past ten years have resulted in the majority of mobile phones being equipped with high quality digital cameras. Every person with a camera phone has the ability to capture and disseminate events happening around them. This, combined with the global popularity of social media, has led to people documenting most aspects of their lives in digital format. In the medical profession this ability has outstripped guidelines regarding the responsible and ethical use of clinical photographs. Recently there have been incidences where patients' photographs have been uploaded onto social media and into the lay press. Arun et al. reported multiple cases where medical students have uploaded bedside clinical pictures onto social media. ${ }^{1}$ A medical doctor from Rhode Island, New York, wrote about one of her patient's injuries on Facebook and even though she did not reveal the patient's name, a third party was able to identify the patient. The doctor was fired from the hospital, reprimanded by the state's medical board and forced to pay a fine. ${ }^{2}$ Another example is that of a runner (who happened to be a medical doctor) who tweeted a picture of himself assisting paramedics with an individual who had collapsed. Part of the individual's face was visible in this picture and was subsequently printed in a newspaper; this caused an outcry as the runner was deemed to be the patient's doctor and bound by patient confidentiality. ${ }^{3}$ The taking of these pictures has become so simple that it can easily go unnoticed by patients and colleagues. This has led to the establishment of 'no photo zones' in certain hospitals, ${ }^{4}$ citing negative outcomes and adverse incidents in order to prevent violation of patient rights and possible litigation.

Despite these negative incidences, photos can be of excellent value as a clinical tool for communication, documentation and improved patient care. In specialities where skin conditions and wounds are managed, clinical photographs form an integral part of good clinical practice. Pictures of open fractures, for example, are an invaluable tool for communication during orthopaedic handover and for consultation with plastic surgeons. As part of the BOAST 4 guidelines, the British Orthopaedic Association suggests that all open fractures should be 
photographed to prevent unnecessary opening of wounds during consultations with the plastic surgeons. ${ }^{5}$ The use of clinical photography must be protected, but clinicians need to adhere to guidelines for its responsible and ethical use. ${ }^{6}$

The intersection between social media and medicine has begun to blur the line between moral conduct as it becomes easier to take and share images on personal devices, and professional responsibility to ensure patient privacy is protected. The aim of this study was to determine the extent of this grey area and suggest ways to clarify the boundaries while protecting a valuable resource.

\section{Materials and methods}

To ascertain the current use of digital photographs in clinical practice, we conducted an online electronic survey among orthopaedic medical officers, registrars and consultants. A survey was generated on an online platform (SurveyMonkey) and a link to the survey was emailed to the South African Orthopaedic Association mailing list and the orthopaedic departments of the universities of Cape Town, Stellenbosch, Bloemfontein, Gauteng (Johannesburg and Pretoria) and KwaZulu-Natal. The survey was conducted over a period of two months and covered three main areas:

1. Scope of respondents' practice and orthopaedic experience

2. The use of clinical photographs for patient care

3. The dissemination and storage of these images

\section{Results}

A total of 150 medical practitioners completed the questionnaire. There were equal numbers of public and private surgeons and $30 \%$ were affiliated with an academic institution. Geographically, 45\% worked in KwaZulu-Natal, 28\% in the Western Cape, $21 \%$ in Gauteng, and the rest were spread over the remaining provinces. Half of the respondents (52\%) had worked in orthopaedics for more than ten years and $29 \%$ between five and ten years. Two-thirds (67\%) were consultants, $22 \%$ were registrars and the remainder $(11 \%)$ were medical officers.

Almost all respondents (99\%) admitted to taking photos of clinical cases; only one consultant and one registrar reported never using photos and both stated this was due to technical reasons. The most frequent use of images was as a communication tool for referrals and handovers $(85 \%)$, followed by record-keeping $(70 \%)$, use as a teaching aid $(57 \%)$ and to monitor clinical progress $(56 \%)$. Close to half $(47 \%)$ of respondents used the photos for research and in publications. Almost two-thirds (61\%) of clinicians had used clinical photos to aide communication with patients. A third $(38 \%)$ of clinicians felt that clinical care would be negatively affected without the use of these images, while $27 \%$ felt that clinical photos made no difference to patient care.
Consent was where the responses varied the most. Most surgeons $(81 \%)$ had obtained written consent previously but did not do it routinely, $6 \%$ stated they always obtained written consent and 13\% had never obtained written consent. Of those who had obtained consent only $20 \%$ (28 respondents) had included permission to show these images to colleagues.

The majority of respondents $(74 \%)$ stored images on their mobile phones, $58 \%$ stored images on their laptops and $26 \%$ on USB flash drives. Sixty-one per cent stated they had secured all the devices used for storing of images with a password or PIN number while 24 respondents $(16 \%)$ had no protection on any of their devices. Two-thirds $(71 \%)$ of respondents stated that no-one else had access to their images while $21 \%$ admitted that family, friends or repair technicians did have access to their images. Three per cent admitted that non-medical people had accessed their clinical photos on a previous occasion. Only $7 \%$ of respondents routinely deleted images after the clinical case was completed; the remainder stored the images for record-keeping (50\%), teaching and presentations (47\%).

Captured images were shared electronically via text or WhatsApp ${ }^{\odot}(58 \%)$ and email $(80 \%)$.

No respondents reported uploading clinical images to Facebook, Twitter, Instagram or Pinterest. Most respondents $(92 \%)$ did not think it would be appropriate to upload these images to a social network, even if anonymised.

Subset analysis revealed that registrars were more likely to password protect their images, take pictures without consent and use images during handover. Forty-five per cent of registrars would delete images once the case is completed while $68 \%$ of consultants kept the images for teaching purposes. Almost half of the registrars (48\%) thought that treating time would be adversely effected if they did not have these images. Consultants were evenly divided as to whether or not photographs make a difference to patient care; one-third (35\%) thought it did make a difference, one-third did not $(33 \%)$ and one-third were undecided. Of those respondents who said that treatment and hospitalisation times would increase and clinical efficiency would decrease, almost half were in state practice $(46 \%)$ with more than ten years' experience (43\%).

\section{Discussion}

South Africa has a heavy trauma burden with a high incidence of road traffic accidents and interpersonal violence. ${ }^{78}$ The care of trauma patients is often made more difficult due to a poor peripheral health service, often manned by relatively junior or inexperienced staff and frequent difficulty in transporting patients from the periphery to the regional referral centres. This can all contribute to patient morbidity. Clinicians in peripheral centres need to be able to triage patients effectively, identifying the differences between those requiring urgent 
transfer and those that can be managed successfully at the base hospitals. Incorrect referrals can consume limited transfer resources and may delay the emergent transfer of appropriate patients. Electronic images and photo sharing can improve patient care by ensuring appropriate referrals and conscientious use of a limited transfer resource and allowing immediate specialist review of injuries. These images also assist with communication across language barriers and lend themselves to efficient documentation for a case record and medico-legal purposes.

Once patients reach their referral centres they often require intradepartmental handover and interdepartmental referral during the course of their management. Digital photography can assist with this communication and limit the need for wound exposure. A recent article by Khanna et al. confirmed the effectiveness of 'WhatsApp' as an intradepartmental communication tool to improve patient-related awareness, communication and handovers. ${ }^{9}$ This research found that clinical images are predominantly used as a handover tool for after-hours admissions. This was closely followed by interdepartmental referrals as is frequently required between orthopaedic surgery and plastic surgery. Images used solely for these purposes should probably be deleted once they have served their purpose.

The storage and dissemination of clinical images beyond their use for patient care is a major ethical concern and warrants specific attention. Images that are not deleted are usually kept for teaching or research purposes. Images saved for these purposes must be accompanied by duly documented informed consent that includes their use in research or publication in peer-reviewed journals. ${ }^{10}$ Taking consent for clinical images was identified as the area that needs the most education and behaviour modification. As a profession this needs to be seen in the same context as ordering special investigations and minor procedures.

The long-term storage of clinical images also needs special consideration. This was one area that our survey identified as being frequently neglected by medical practitioners. Photographs saved on smartphones should be downloaded or moved onto a safer storage device as soon as possible such as password protected desktops and laptop computers and secure external hard drives. The use of USB flash drives to save images should be discouraged as these devices have the greatest potential to be misplaced and accessed by unintended individuals.

Digital photography can allow doctors to work more effectively and efficiently. It allows easy and instantaneous documentation for communication and record-keeping. To continue using this resource, doctors need to regulate themselves as a profession with regard to the use of digital photography in their daily clinical work. Images should be stored in a secure manner to ensure that they are not accessible to the general public and these images should never be shared via insecure means or uploaded onto social media. Consent needs to be obtained and documented appropriately in the patient's notes or file.
The greatest limitation of this study stems from its survey design. Most respondents reported no major ethical breaches with their use of clinical photography. This could be due to the fact that surgeons who do not follow strict control with clinical images were more likely to decline to complete the survey.

Future research should focus on newer software that is becoming available. The Figure1 application ${ }^{11}$ is designed to allow sharing of pictures between medical professionals to enable 'brain storming' of difficult cases. This software is a free photo sharing app for healthcare professionals and is available on iOS and Android. All images are reviewed by a medical officer and team of moderators. They verify that all identifying information has been properly removed from each image before it is uploaded.

\section{Recommendations}

While the formulation of a set of guidelines is beyond the scope of this paper, it would be prudent for the medical profession to adhere to basic recommendations regarding the use of digital images in clinical practice.

- Consent: Obtain and document consent for all clinical photos ensuring that all future uses of the photos have been defined in the consent form.

- Storage: Store and protect images on passwordprotected devices. Delete images when no longer needed.

- Dissemination: Share images only when clinically relevant and use encrypted services where possible. Refrain from uploading clinical images onto social media sites.

\section{Conclusions}

Digital photography is now at the fingertips of anyone with a modern mobile phone. Although the use of pictures may aid in patient care, the inappropriate storage and unethical dissemination of these images should be guarded against. It is imperative that consent be obtained and documented for each case where pictures are taken. Demonstrating this self-regulation to the relevant regulatory bodies would be beneficial in order to avoid directives that would prevent us from using these images as has been the trend internationally.

\section{Conflict of interest statement}

Ethical approval for conducting this research was obtained from the UKZN Biomedical Research Ethics Committee and all responses were anonymised.

The content of this article is the original work of the authors. No benefit of any form has been received or will be received from a commercial party related directly or indirectly to the subject of this article. 


\section{References}

1. Arun BT, Sharmila V. Photographing patients: an emerging unethical trend Indian J Med Ethics. 2011;8(2).

2. ER Doc Forgets Patient Info is Private, Gets Fired for Facebook Overshare. http:/ / abovethelaw.com/2011/04/ er-doc-forgets-patient-info-is-private-gets-fired-forfacebook-overshare/ (accessed November 2014).

3. The ethical dilemma of being a runner, doctor and journalist. http://www.kevinmd.com/blog/2014/05/ ethical-dilemma-runner-doctor-journalist.html (accessed November 2014).

4. http:// med.stanford.edu/shs/update/archives/FEB2011 / cellphone.htm (accessed November 2014).

5. Cell phone photos are easy, maybe too easy, to take - check rules before shooting patients http:/ / www.bapras.org.uk / docs / default-source / default-document-library / bapras_boa-lower-limb-standards-read-only.pdf (accessed November 2014).

6. Mutalik S. Digital Clinical Photography: Practical Tips. J Cutan Aesthet Surg. 2010;3(1):48-51.

7. Norman R, Matzopoulos R, Groenewald P, Bradshaw D. The high burden of injuries in South Africa. Bulletin of the World Health Organization 2007;85(9):649-732 (PMCID: PMC2890139).

8. Parkinson F, Kent S, Aldous C, Oosthuizen G, Clarke D. Road traffic crashes in South Africa: the burden of injury to a regional trauma centre. S Afr Med J. 2013;103(11):8502. doi:10.7196/samj.6914.

9. Khanna V, Sambandam S, Gul A, Mounasamy V. "WhatsApp"ening in orthopedic care: a concise report from a 300-bedded tertiary care teaching center. Eur J Orthop Surg Traumatol. 10 Jan 2015.

10. Kunde L, McMeniman E, Parker M. 'Clinical photography in dermatology: ethical and medico-legal considerations in the age of digital and smartphone technology.' Australas J Dermatol. 2013;54(3):192-97.

11. Free, safe photo-sharing for health professionals. https:/ / figure1.com (Accessed 15 February 2015).

This article is also available online on the SAOA website (www.saoa.org.za) and the SciELO website (www.scielo.org.za). Follow the directions on the Contents page of this journal to access it. 